\section{IN BRIEF}

\section{$\Rightarrow$ PROSTATE CANCER}

\section{Immunogenomic landscape of dMMR tumours}

Immunogenomic analysis of advanced prostate cancer has identified immunological alterations associated with defective mismatch repair (dMMR) that could inform immunotherapy strategies. Overall, dMMR status was confirmed in $8.1 \%$ of patients and was associated with poor overall survival; dMMR tumours with high microsatellite instability (MSI) had increased mutational load, T cell infiltration, and PD-L1 expression. Interestingly, dMMR mutational signatures positively correlated with MSI and expression of immune-associated genes, including PDL1 and PDL2.

ORIGINAL ARTICLE Nava Rodrigues, D. et al. Immunogenomic analyses associate immunological alterations with mismatch repair defects in prostate cancer. J. Clin. Invest. https://doi.org/10.1172/JCl121924 (2018)

\section{IMAGING}

\section{${ }^{18} \mathrm{~F}$-Fluciclovine-PET-CT LOCATEs recurrence}

The LOCATE trial reports the influence of ${ }^{18} \mathrm{~F}$-fluciclovine$\mathrm{PET}-\mathrm{CT}$ on the management of men with biochemical recurrence of prostate cancer. The study prospectively enrolled 213 men with rising PSA levels but negative or equivocal findings on standard-of-care imaging after curative intent treatment. ${ }^{18} \mathrm{~F}$-Fluciclovine-avid lesions were detected in 122 (57\%) patients and management plans were changed in 126 patients (59\%), of which 98 (78\%) were major changes in treatment modality and $88(70 \%)$ were informed by positive findings on ${ }^{18}$ F-fluciclovine-PET-CT.

ORIGINAL ARTICLE Andriole, G. L. et al. The impact of positron emission tomography with ${ }^{18} \mathrm{~F}$-fluciclovine on the management of patients with biochemical recurrence of prostate cancer: results from the LOCATE trial.J. Urol. https://doi.org/10.1016/ j.juro.2018.08.050 (2018)

\section{KIDNEY CANCER}

\section{CXCR4-HIF-1 $\alpha$ interaction drives metastasis}

New research offers mechanistic and clinical insights into CXC chemokine receptor 4 (CXCR4) nuclear localization in renal cell carcinoma (RCC). CXCR4 nuclear localization - which frequently occurred in RCCs, particularly metastases - was associated with poor prognosis and induced metastasis in vitro and in vivo. A mechanistic feedforward loop was identified whereby nuclear CXCR4 promoted hypoxia-inducible factor $1 \alpha(\mathrm{HIF}-1 \alpha)$ accumulation, which, in turn, induced CXCR4 transcription. Clinically, CXCR4 and HIF-1a subcellular expression levels were associated with poor prognosis independently and when combined with TNM stage.

ORIGINAL ARTICLE Bao, Y. et al. A feed-forward loop between nuclear translocation of CXCR4 and HIF-1a promotes renal cell carcinoma metastasis. Oncogene https://doi.org/ 10.1038/s41388-018-0452-4 (2018)

\section{PROSTATE CANCER}

\section{Interfocal genomic heterogeneity uncovered}

A large-scale study has for the first time reported the high degree of interfocal genomic heterogeneity in multifocal primary prostate cancer, which could inform clinical decisionmaking. Whole-exome sequencing in 89 tumour foci from 41 patients revealed that $76 \%$ of matched foci from the same prostatectomy specimen had no common somatic point mutations and that DNA copy number alterations were rarely shared among foci. Although a small number of mutations were shared across foci, they rarely occurred in driver genes. ORIGINAL ARTICLE Løvf, M. et al. Multifocal primary prostate cancer exhibits high degree of genomic heterogeneity. Eur. Urol. https://doi.org/10.1016/j.eururo.2018.08.009 (2018)

\title{
Correlates of alisertib response
}

New therapies are urgently needed for metastatic neuroendocrine prostate cancer (NEPC), an aggressive variant that can evade androgen receptor (AR)targeted therapies. N-MYC and Aurora kinase A are overexpressed in NEPC and are, therefore, putative targets. Newly reported findings from a phase II study now suggest that only a subset of patients with NEPC derive benefit from the Aurora kinase A inhibitor alisertib.

The single-arm phase II trial investigated the efficacy of single-agent alisertib (50 mg twice daily) in 60 men with metastatic castration-resistant prostate cancer (CRPC). Eligibility criteria included $\geq 1$ investigator-defined NEPC feature (NEPC, $n=45$ ) and, for those without NEPC features, liver metastasis in the absence of PSA progression (CRPC-Adeno, $n=15)$. The primary end point was 6-month radiographic-progressionfree survival (rPFS) and secondary end points included response rate, overall survival (OS) and toxicity. Mandatory pretreatment biopsy samples were also analysed to investigate the pathological and molecular correlates of clinical response to alisertib.

The 6-month rPFS was $13.4 \%$ overall $(16.7 \%$ and $5.3 \%$ in the NEPC and CRPC-Adeno groups, respectively); thus, the trial did not meet its primary end point. The objective response rate was $3.3 \%$ and median OS was 9.5 months, with no significant differences between groups. Interestingly, four exceptional responders were identified - two patients with complete resolution of liver metastases and two with prolonged stable disease whose tumours underwent further evaluation.

Genomic profiling and pathological evaluation of pretreatment metastatic biopsies revealed that only $24 \%$ of evaluable cases harboured AURKA and/or $M Y C N$ amplification. Across the cohort, AURKA amplification was associated with improved OS but not PFS. Interestingly, although MYCN amplification was not associated with clinical outcomes, tumours from the exceptional responders harboured molecular evidence of aberrant N-MYC signalling (concurrent MYCN and AURKA amplification or overexpression). Organoids established from NEPC pretreatment biopsy samples derived from an exceptional responder and a nonresponder recapitulated clinical responses to alisertib. Further characterization using a proximal ligation assay revealed that alisertib could only disrupt the N-MYC-Aurora kinase A complex in responder organoids despite high baseline expression, suggesting the potential of this assay to act as a functional readout of alisertib response.

Overall, the trial did not meet its primary end point, but exceptional responders and molecular correlates of alisertib response were identified, which could inform future studies.

"This study improves our understanding of the clinical and molecular features of this aggressive and often under-recognized subgroup of advanced prostate cancer; additional studies focused on NEPC and targeting N-MYC-Aurora kinase A are warranted," concludes author Himisha Beltran.

Conor A. Bradley

ORIGINAL ARTICLE Beltran, H. et al. A phase II trial of the aurora kinase $A$ inhibitor alisertib for patients with castration resistant and neuroendocrine prostate cancer: efficacy and biomarkers. Clin. Cancer Res. https://doi.org/ 10.1158/1078-0432.CCR-18-1912 (2018) 